\title{
TREATMENT OF NEO-VASCULARIZATION WITH OXYGEN AT HIGH PRESSURE*
}

\author{
BY \\ JOHN WINSTANLEY \\ London
}

THE hope that the proliferating new vessels, which so often complicate diabetic retinopathy, might be influenced by ambient hyperoxia was based upon the following grounds:

(1) It has long been known that the vessels of the central nervous system, including those of the retina, are sensitive to the ambient oxygen concentration. Cusick, Benson, and Boothby (1940) were able to show that there was a considerable reduction in the calibre of the retinal vessels in the normal subject who breathed pure oxygen for short periods, and Sieker and Hickam (1953) have shown that, while this response can still be elicited in the presence of diabetic retinopathy, the extent of the calibre changes is much reduced.

(2) Ashton, Ward, and Serpell (1953) in classical experiments in their researches into the cause of retrolental fibroplasia, first demonstrated the obliterative influence of a high ambient oxygen concentration upon the developing vessels of the immature retina. Moreover Ashton went on to show that this phenomenon is confined to the growing immature retinal vessels, and does not occur in the other immature vessels of the eye, nor in the vessels of the mature retina.

Ashton put forward the suggestion that a trial should be made of the effect of ambient hyperoxia upon the proliferating retinal vessels of the diabetic eye, and it is the purpose of this short paper to report such a trial, which was carried out in the belief that the ability to influence the new-formed vessels in this way might ultimately form the basis of a practical form of therapy.

The mechanism of the vaso-obliterative response of immature retinal vessels to ambient hyperoxia is still obscure, even in experimental animals in which the process has been well established and often repeated, so that no help could be obtained from this source as to the site or type of retinopathic vessel most likely to respond.

In practice, the vessels chosen for the closest scrutiny were those budding capillaries lying on the advancing edge of a so-called rete mirabile, the assumption being that these vessels were those most likely, to possess the same qualities of immaturity as those of the developing kitten or rabbit.

\footnotetext{
* Paper given at the joint meeting of the Irish Ophthalmological Society and the British Diabetic Association held at Dublin on April 25 to $27,1963$.
} 
It is only in quite recent work involving the use of the electron microscope and techniques of retinal digestion that Ashton and Pedler (1962) have been able to demonstrate certain histological features which distinguish the mature from the immature retinal vessel, and, so far as I am aware, this work has not yet been applied to the proliferating vessels of diabetic retinopathy. The assumption that the vessels are immature rests on the clinical evidence that they can be seen to be actively growing.

Again, animal experiments have not shown that proliferating capillaries outside the confines of the retina share in the vaso-obliterative response to hyperoxia, and it has been suggested that only the vessels contained within the retina will be so affected.

On the other hand Ashton has recently shown that the developing retinal vessels of the rabbit, which lie freely on the surface of the retina, react to ambient hyperoxia in a manner closely similar to those of the kitten which lie within the retina.

It would therefore seem reasonable to expect that correspondingly situated developing new vessels in diabetic retinopathy might also behave in a similar manner.

In planning these tests two other important facts which emerged from the animal experiments were made use of.

First, Pedler (1961) showed that the partial closure of the developing retinal vessels of the ratling subjected to ambient hyperoxia at atmospheric pressure was converted to total obliteration when the ambient oxygen pressure was raised by one atmosphere.

Secondly, in their animal experiments, Ashton and Pedler (1962) found that the earliest vaso-obliterative changes were to be found after 6 hours' exposure to hyperoxia at atmospheric pressure.

It seems, then, that the response of the immature retinal vessel to ambient oxygen depends upon its concentration, its pressure, and the duration of exposure.

It was therefore decided to test the sensitivity of the proliferating vessels of diabetic retinopathy in response to the maximum safe stimulus of hyperoxia. At once we were in some difficulty, for the toxic effects of hyperoxia under pressure are known not only to be severe, but also to become manifest after different degrees of exposure in different subjects, and even in the same subject at different times. So that, as the work of Donald (1947) has shown, there is no clear-cut demarcation between an innocuous and a toxic exposure.

In this respect, however, I acknowledge gratefully the help of my colleague, Dr. Charles Foster, who has given invaluable advice as to safety margins based upon his extensive experience of the management of patients undergoing radiotherapy in an oxygen compression chamber. 
The Table shows the combination of oxygen concentration, pressure, and duration of exposure used, and represents in each case what was considered to be the maximum safe stimulus. The concentration was the highest that could be obtained, and was determined by gas analysis from the breathing circuit during the tests.

TABLE

\begin{tabular}{c|c|c}
\hline $\begin{array}{c}\text { Oxygen Chamber Pressure } \\
\text { (lb/in.) }\end{array}$ & Duration (hrs) & $\begin{array}{c}\text { Oxygen Concentration } \\
\text { (per cent.) }\end{array}$ \\
\hline 15 & 6 & 97 \\
20 & 3 & 97 \\
30 & 2 & 97 \\
\hline
\end{tabular}

After certain preliminary trials, three patients were selected to undergo the tests, all had advanced diabetic retinopathy, with easily observable proliferating new vessels in the fundus of the eye (Fig. 1).

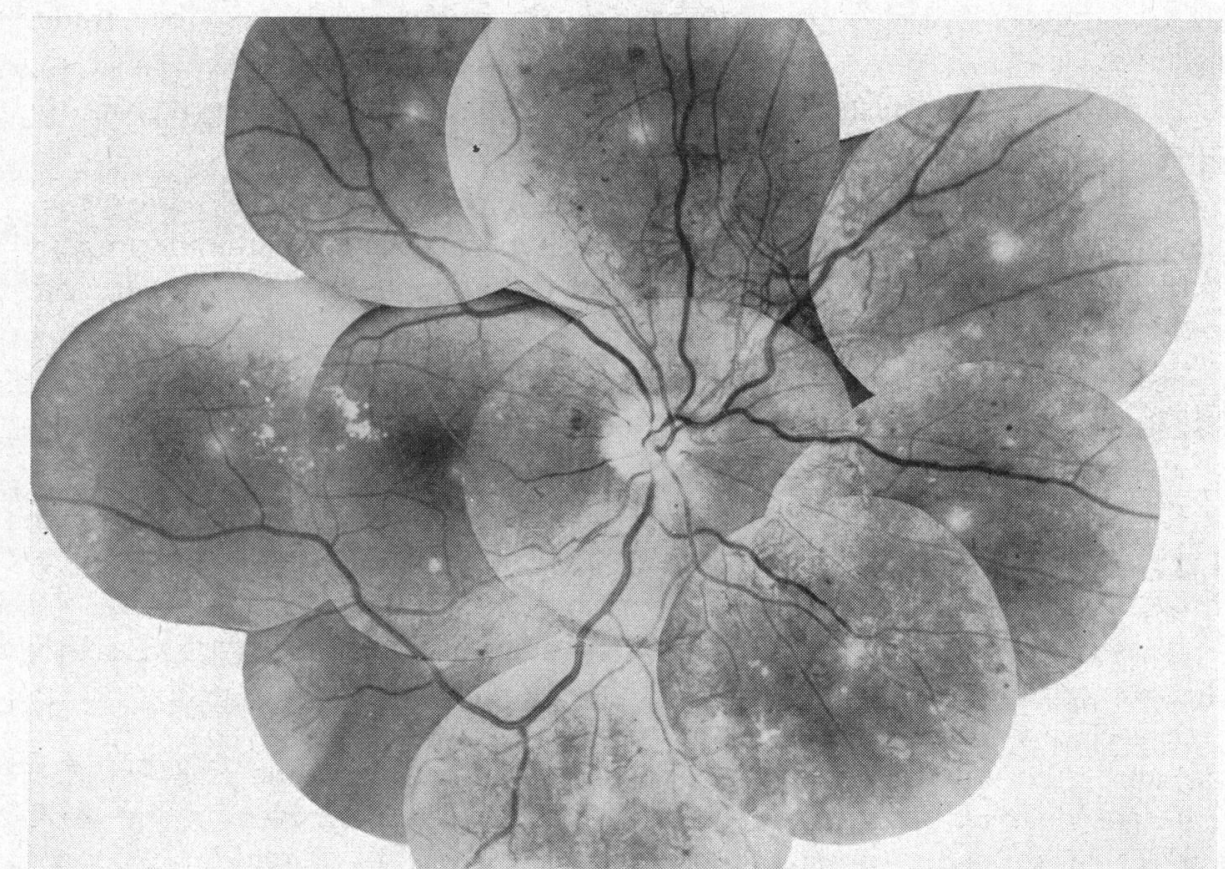

FIG. 1.-Vasoproliferative diabetic retinopathy.

So that the observer might see the fundus vessels with the direct ophthalmoscope during the test, it was necessary to use a compression chamber large enough to accommodate both the patient and the observer, but the large volume of oxygen under pressure thus required would be both expensive and dangerous from the risk of ignition. The compression was therefore carried 
out in an air-filled chamber, the patient breathing pure oxygen administered through a closed circuit* (Figs 2 and 3 ).

Fig. 2.-Oxygen chamber; the size can be gauged by that of the chair and table just visible inside the chamber.
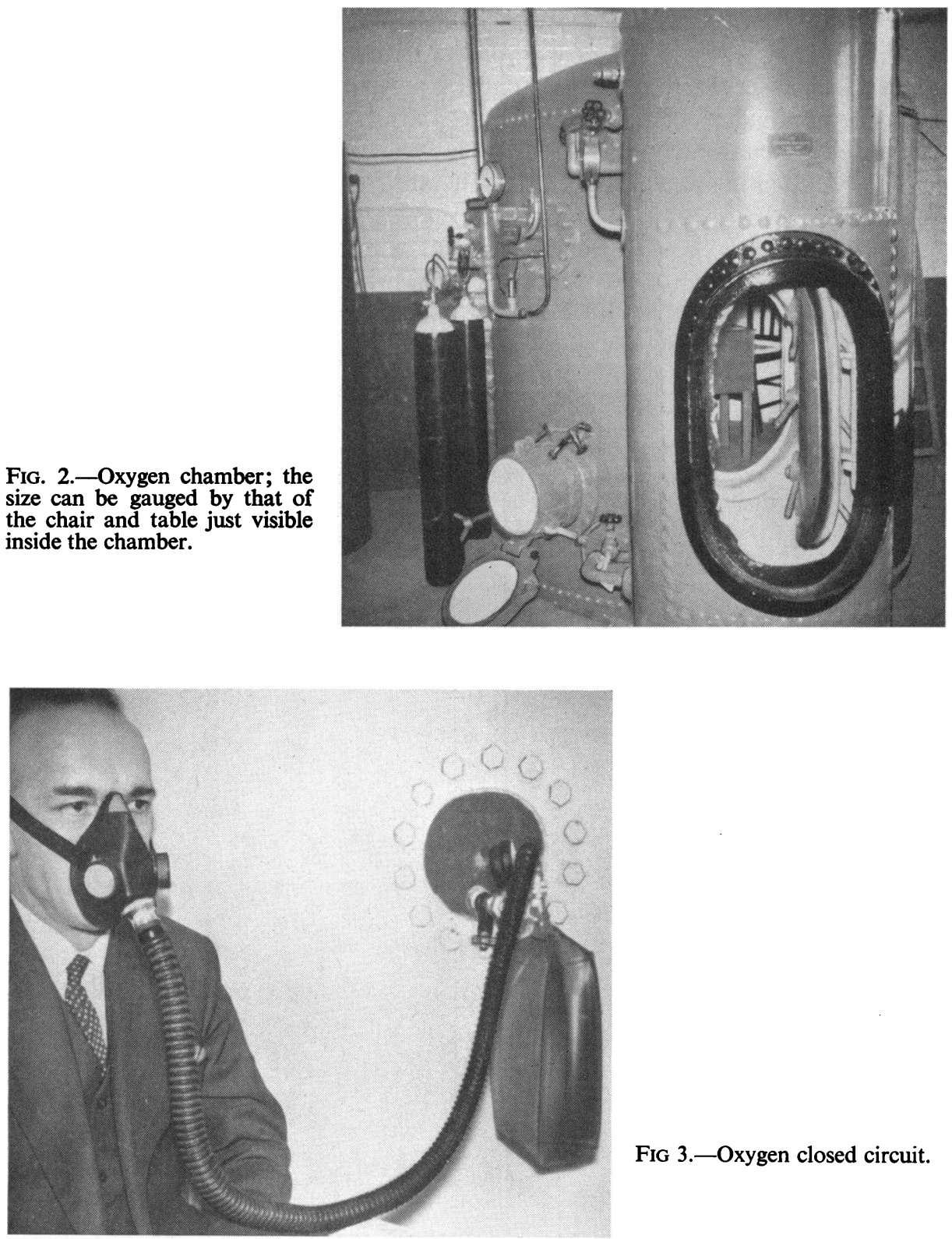

FIG 3.-Oxygen closed circuit.

* This compression chamber and oxygen circuit, with all the technical assistance necessary for its use, was put at our disposal with characteristic generosity by Sir Robert Davis at the diving school of Messrs. Siebe Gorman. 
Eight tests were made under the conditions described, but in no case was any change observed in any of the vessels visible with the ophthalmoscope, either during or immediately after the tests. While it is not possible, without microscopy, to be certain that no change occurred in the calibre of the vessels, it is possible to state from the ophthalmoscopic appearances, that all the vessels observed remained patent throughout the tests. Nor were there any haemorrhagic episodes immediately after the tests, which might have suggested a reactionary dilatation.

The conclusion seems justified that the proliferating new vessels of diabetic retinopathy are insensitive to ambient hyperoxia in non-toxic doses. It is right to point out however, that in these tests the capillaries, both normal and abnormal, which lie within the retina have not been visible within the ophthalmoscope, so that, if it were possible, further tests should be carried out with the fluorescein technique to study the patency of the retinal capillaries themselves.

I should like to acknowledge the invaluable help of my colleague Dr. Charles Foster. It is also a pleasure to express my gratitude to Sir Robert Davis and the Staff of the Diving School at Messrs. Siebe Gorman Ltd. without whose help this work could not have been carried out.

\section{REFERENCES}

Ashton, N. (1960). Personal communication. (1961). Trans. ophthal. Soc. U.K., 81, 145. and Pedler, C. (1962). Brit. J. Ophthal., 46, 257. WARD, B., and SerPell, G. (1953). Ibid., 37, 513.

Cusick, P. L., Benson, O. O., and Boothby, W. M. (1940). Proc. Mayo. Clin., 15, 500. DONALD, K. W. (1947). Brit. med.J., 1, 667, 712 .

Pedler, C., (1961). Quoted by Ashton (1961).

SIEKER, H. O., and HICKAM, J. B. (1953). Circulation, 7, 79. 